\title{
Mooring a Space Station: Media Infrastructure and the Inhuman Environment
}

\author{
Katarina Damjanov and David Crouch
}

Among various ventures to extend earthly activities into Earth's inhuman exterior, space stations occupy a special place. Designed to provide inhabitable enclosures, their operations highlight the mediatic capacity of infrastructures to condition our environmental circumstances. Media infrastructures have long been integral to the development of human settlements (Mattern 2015) and their potential to interfere with our material and social relations is more advanced in the setting of outer space, where their complex technological and organisational edifice must be mobilised to sustain human ways of life (Damjanov 2015). Space stations in this sense constitute media infrastructures par excellence. They envelop complex configurations of the technological moorings and data-gathering, storing and relaying capacities of media, at once embodying a range of the structural groundings and operative logics of media infrastructures, and protracting them into a novel environmental situation. Such arrangements provide a testing ground for probing into the limits and possibilities of our infrastructural lives, offering a condensation of their socio-technical features and effects, but also an expansion of the problematics that surround their assertion and control. 
Several stations have occupied Earth's low orbit thus far, for various lengths of time, from the Soviet Union's Salyut 1 (1971) and the United States' Skylab (1973-79) to Mir (1986-2001) and the International Space Station (ISS, 1998-). Their historical development marks advances in human infrastructural endeavour - each subsequent station providing a more sophisticated and durable dwelling environment. Currently, the ISS is the sole such settlement in space, having been continuously occupied since the arrival of the first crew. A joint project between a number of space agencies and various scientific, commercial and military interests, it constitutes unique infrastructural entanglements involving an orbital choreography of still-malleable socio-technical systems and their political, economic and cultural scaffolding. The ISS is designed to provide a safe harbour amid its unforgiving surroundings, necessitating experimental approaches to upholding the courses of life as we know it, but at the same time demanding their highly regimented distribution and arrangement (Damjanov and Crouch 2019). A scripting of media infrastructures in progress with the capacity to further assert their high-tech systems and processes, the ISS encapsulates a host of strategies intended to upend our environmental confines.

The International Space Station.

Credit: https://www.

nasa.gov.

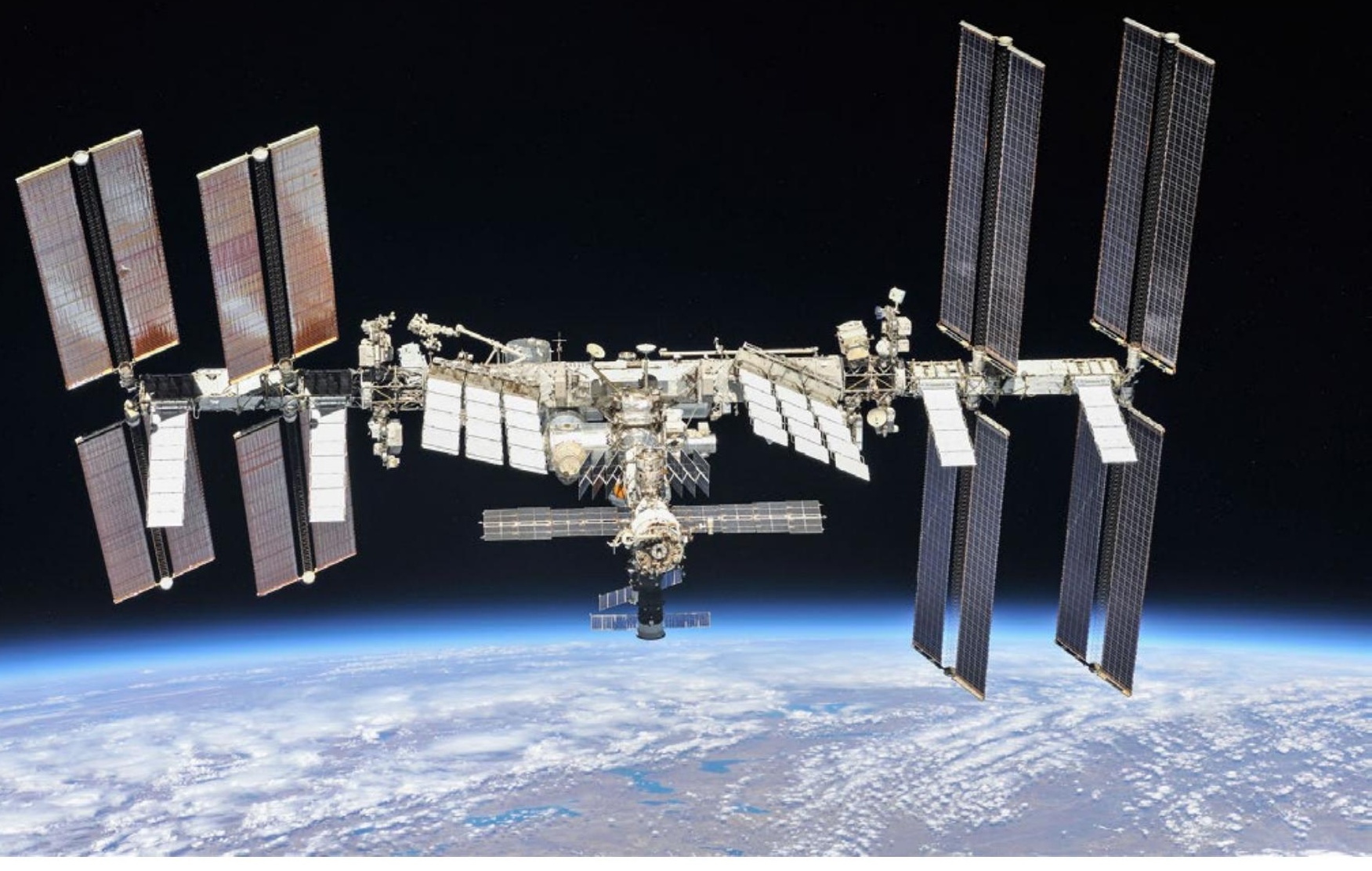


The ISS features an intricate infrastructural configuration. Its modular architecture comprises two main sections operated respectively by the United States' National Aeronautics and Space Administration (NASA) and Russia's Roscosmos, and interconnected through an Environmental Control and Life Support System that conducts multiple subsystems, from sanitation facilities to atmosphere control systems. The whole station is strapped together with "eight miles of wire" that links an electrical power system generated by solar arrays (NASA 2019). The American section is maintained by "more than 1.5 million lines of flight software code run on 44 computers communicating via 100 data networks transferring 400,000 signals" (NASA 2019). It sends transmissions to ground stations via the Tracking and Data Relay Satellite System, while communication between nodes is conducted through an internal wireless network. The airlocks and arrays, docking ports and cargo bays, laboratories and cupolas all form a sealed and pressurised habitat that conditions life in a casing of software and hardware. It has thus far been occupied by over two hundred astronauts, several space tourists, various plants and animals and a range of assistive technological apparatus, all working in tandem to facilitate trials across the domains of science - from physics, the geosciences, astronomy and robotics, to chemistry, biology, medicine and meteorology. The ISS encloses all of its components, human and non-human, biotic and abiotic, within an intricate ecology reliant on the design of its interlocking life support systems, recasting a gamut of both "natural" and "artificial" terrestrial processes in purely infrastructural terms.

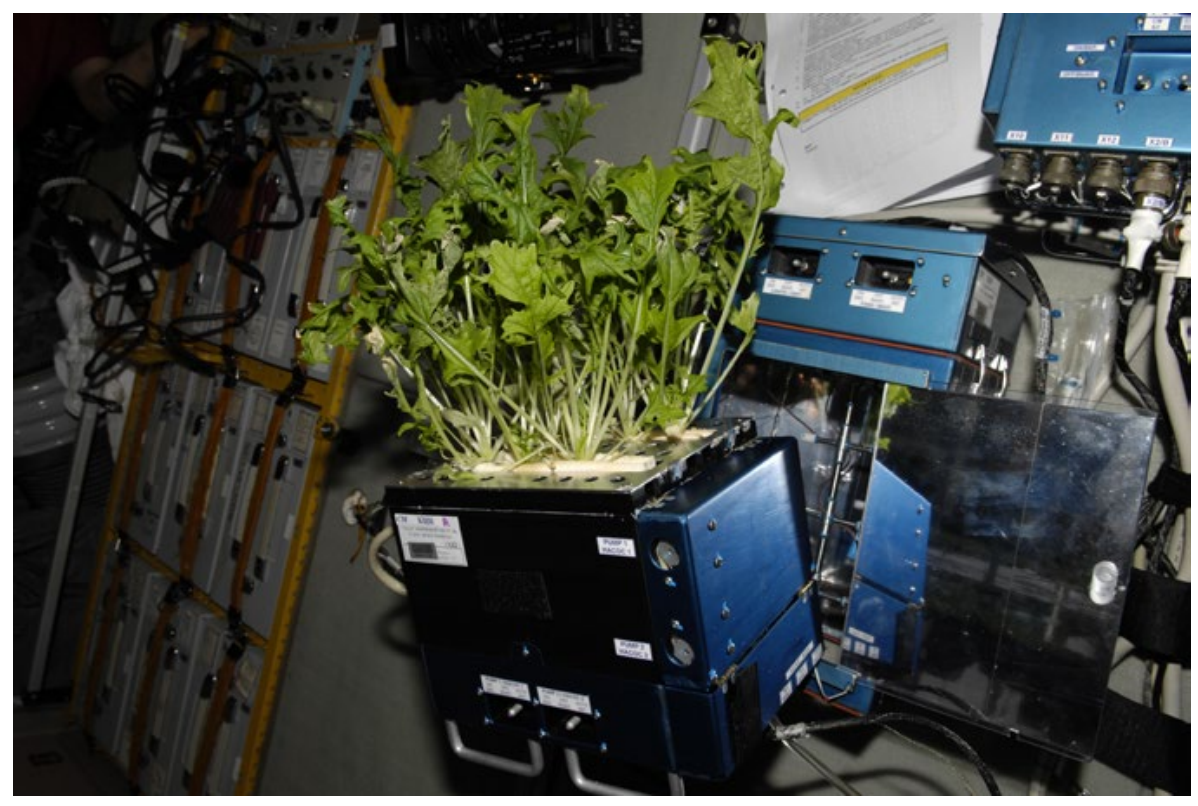

The ISS itself relies on continued assistance from the world below. Work and life aboard are contingent upon the station's terrestrial moorings. Most essential resources are delivered - food, air, water, various tools and materials, instruments and specimens, from toilet paper to solar panels, are all supplied every few months at high cost (NASA 2018). Seemingly separated from Earth, the ISS is in fact closely interlinked with the planet through the signal traffic between it and its ground controls. The very
Edible plants ready for harvest. Credit: https://www. nasa.gov. 
materiality of these signal exchanges establishes underlying structures of connection and direction. All activities at the ISS are continually monitored through multiple forms of scrutiny, which include comprehensive details about the status and operations of all its systems and subsystems, including the biometric data of its inhabitants - as NASA describes: "on-orbit software" and "approximately 350,000 sensors" monitor "crew health and safety" (NASA 2019). The station's modular segments, robotic arms, living quarters and experiment bays are all part of an architecture that constantly collects and distributes data, evidence and information. It is this intense, all-encompassing mediation that shapes and gives form to life in orbit. Highlighting the infrastructural capacities of "technical networks" to create "communicative spaces" (Mattern 2015: 98), the ISS ecosystem establishes a unique social space that also maintains forms of contact with both its terrestrial and orbital exterior. As media infrastructure, it is in this sense a multilayered orchestration of "communication and communion" (Mattern 2015: 95), which enables the station's connectedness, but also its thorough control.

The hermetic enclosure, containment and rigorous observation of the ISS cultivate particular infrastructural responses to the strategic management and organisation of human settlements in extreme environments. Innovative technologies are regularly recruited to assist in developing more amenable and efficient day-to-day operations - such as the dexterous humanoid robot, Robonaut, which has worked alongside crews since 2012 (Badger 2019). Simultaneously, continuous improvements are made towards securing the station's greater autonomy - in 2014, a 3D printer was brought to the station to enable astronauts to manufacture small tools and hardware rather than wait for them to be dispatched (NASA 2014).

An astronaut and Robonaut work together. Credit: https://www. nasa.gov.

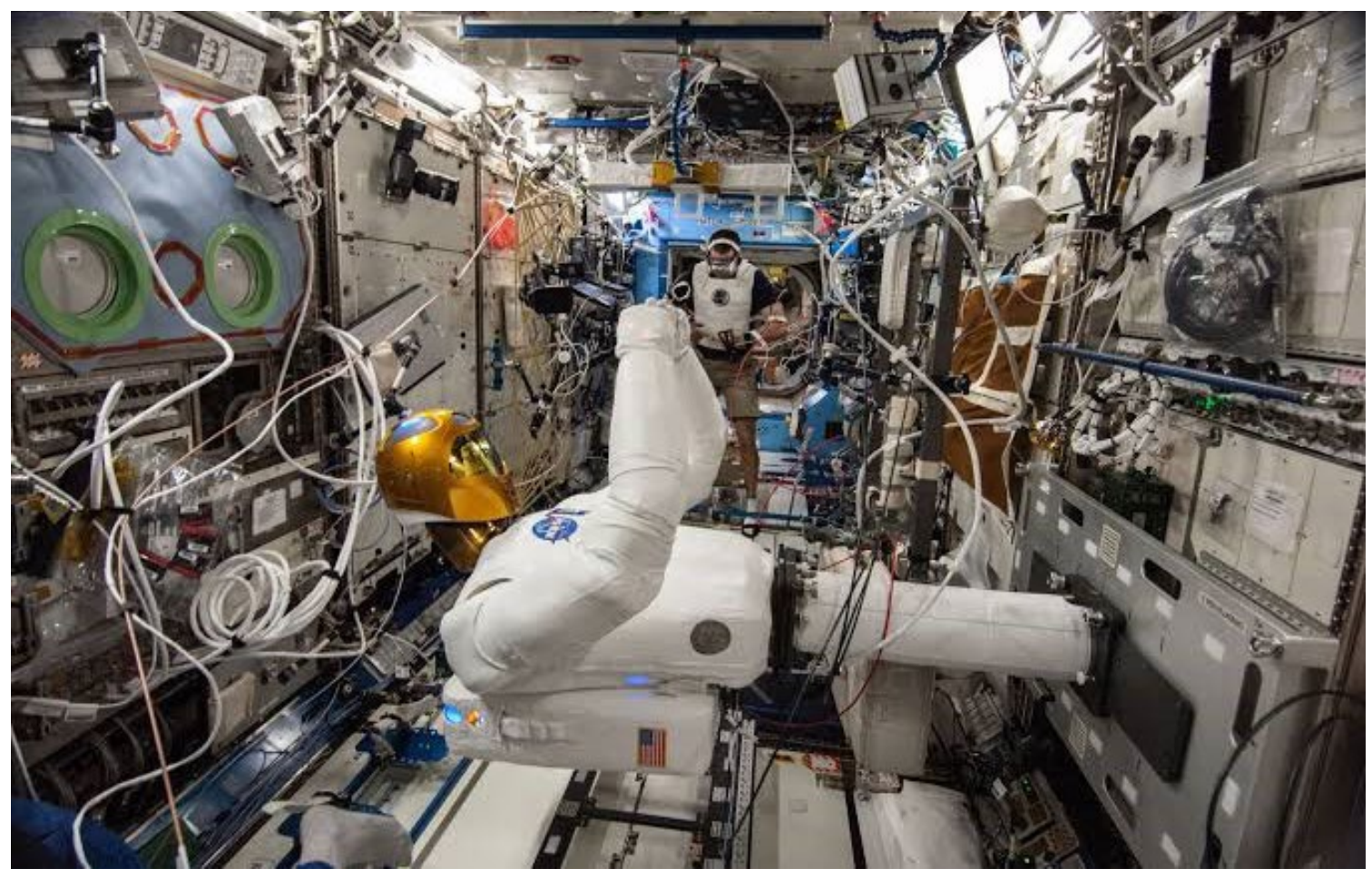


At the same time, better connections with the planet below are established; in 2019, NASA upgraded the internet communication systems with the station, achieving speeds greater than on Earth (Peters 2019). Organised and operated as a multifaceted media infrastructure, the ISS becomes both increasingly self-sustaining, but also systematically conditioned.

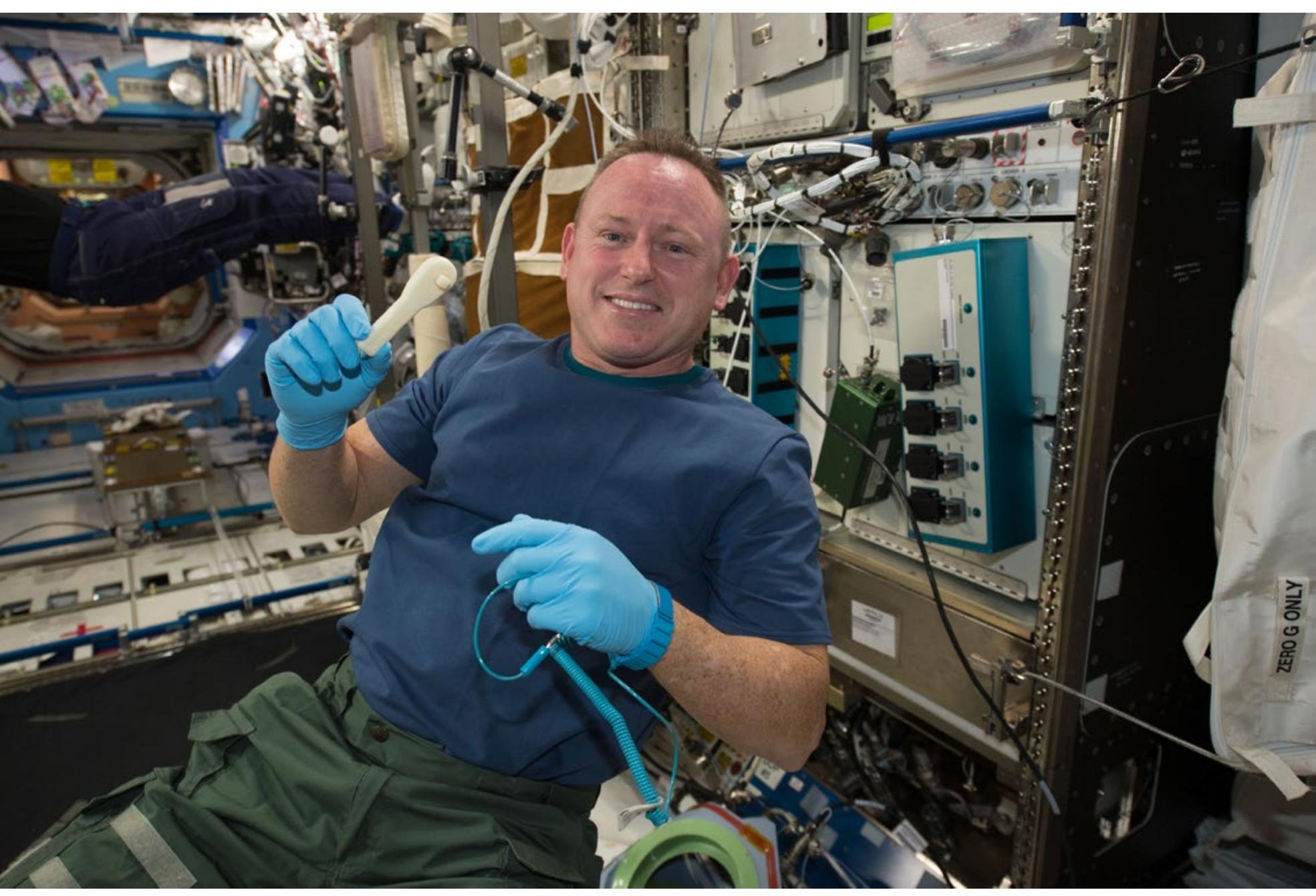

These provisions are not purely technical; they also involve particular political and cultural economies and agendas. Based around an idea of international cooperation in the commons of outer space, the ISS suggests successful connection and exchange, but also various kinds of social restriction and all-inclusive interference. The proceedings of everyday life aboard the station are video-streamed in real time to global audiences, while actually accessing the station is difficult; very few humans qualify for residence, only particular nations participate, only selected projects and experiments are permitted and only certain technologies recruited to assist (Damjanov and Crouch 2019). Restrictions and regulations abound - from the chemical composition of objects, to the health of biological subjects. On the other hand, the station captures planetary attention;
An astronaut holds a 3D-printed wrench. Credit: https://www. nasa.gov. 
sometimes visible with the naked eye, it appeals to the collective imagination as a revolving symbol of human progress in space. Entangled with Earth, the ISS reproduces all the tensions that undergird the terrestrial settlements of the species - its hierarchies, exclusions and uneven exchanges - but juxtaposes these earthly problems in unusual ways, condensing and refracting them amid the inhuman environment of space. As media infrastructure, it connects these experimentations and rearrangements with life on Earth, opening up social and material traffic that continues to reshape human techno-environmental relations.

The ISS, however, will not last forever - beyond the station's eventual decommissioning, its fate is uncertain. Boeing is currently contracted to maintain the hardware until 2028 (Maass 2015), and in 2018 the United States passed the Leading Human Spaceflight Act, intending to prolong the activities aboard until 2030 (Smith 2018). Roscosmos and NASA have each considered the station's afterlife. Parts of the Russian segment are proposed as the groundwork for another piloted complex, forming "the core of a new orbital outpost, which would serve as a haven and assembly shop for deep space missions heading to the Moon, Mars and beyond" (Zak 2009). In 2011 Boeing suggested using leftover ISS hardware to construct an "Exploration Gateway Platform" that would contribute to "long duration habitat evolution" and provide a "flexible path to exploration" (Raftery 2011). Such plans for off-Earth settlement are both highly speculative and already in motion, and perhaps made more pressing by the spectre of planetary precarity. Offering the foundational steps and systems needed to maintain life in space, the ISS may play a pivotal role in the "media-infrastructural historiography" (Mattern 2015: 105). It constitutes a significant new form in the ongoing development of complex infrastructure, highly networked systems and the corresponding formation of human social, political and cultural milieus. As both an experiment in life at the edge and also a platform from which further to advance the expansion of its infrastructural logics, the "groundwork" of the ISS continues to concentrate and "uplift" human prospects in space.

\section{References:}

Badger, Julia. 2019. “Robonaut." Available at: https://robonaut.jsc.nasa.gov/R2/.

Damjanov, Katarina. 2015. "The Matter of Media in Outer Space: Technologies of Cosmobiopolitics." Environment and Planning D: Society and Space 33 (5): 889-906. DOI: https://doi.org/10.1177/0263775815604920.

Damjanov, Katarina and David Crouch. 2019. "Orbital Life on the International Space Station." Space and Culture 22 (1): 77-89. DOI: https://doi.org/10.1177/1206331217752621.

Mattern, Shannon. 2015. "Deep Time of Media Infrastructure." In Signal Traffic: Critical Studies of Media Infrastructures, edited by Lisa Parks and Nicole Starosielski, 94-112. Urbana, Chicago and Springfield: University of Illinois Press. 
NASA. 2014. “International Space Station's 3-D Printer." Available at: https:// www.nasa. gov/content/international-space-station-s-3-d-printer.

NASA. 2018. "What Does It Take to Keep the Station Stocked With Supplies?" Available at: https://blogs.nasa.gov/spacestation/2018/04/30/what-does-it-take-to-keep-thestation-stocked-with-supplies/.

NASA. 2019. "International Space Station Facts and Figures." Available at: https://www. nasa.gov/feature/facts-and-figures.

Maass, Ryan. 2015. "NASA Extends Boeing Contract for International Space Station." Space Daily, 30 September. Available at: http://www.spacedaily.com/reports/NASA extends_Boeing_contract_for_International_Space_Station_999.html.

Peters, Matthew D. 2019. "Data Rate Increase on the International Space Station Supports Future Exploration." Available at: https://www.nasa.gov/feature/goddard/2019/datarate-increase-on-the-international-space-station-supports-future-exploration/.

Raftery, Michael. 2011. “ISS Exploration Platform Concept." Available at: https://www. nasa.gov/pdf/604659main_6\%20-\%20Panel\%203_Raftery_Final.pdf.

Smith, Marcia. 2018. "Babin Introduces Leading Human Spaceflight Act." Space Policy Online, 26 September. Available at: https://spacepolicyonline.com/news/babinintroduces-leading-human-spaceflight-act/.

Zak, Anatoly. 2009. "Russia 'to save its ISS modules'." BBC News, 22 May. Available at: http://news.bbc.co.uk/2/hi/science/nature/8064060.stm.

Cite as: Damjanov, Katarina and David Crouch. 2020. "Mooring a Space Station: Media Infrastructure and the Inhuman Environment." Roadsides 3: 7-14. DOI: https://doi. org/10.26034/roadsides-202000302. 


\section{Authors:}
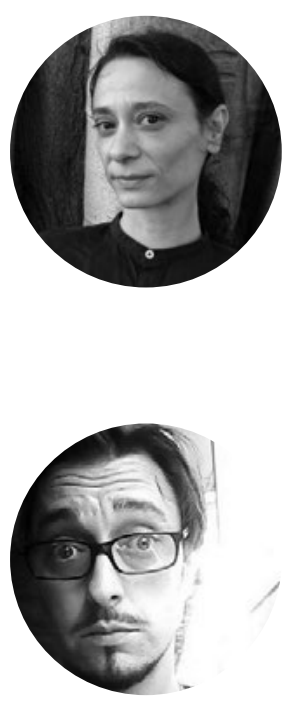

Katarina Damjanov teaches in the School of Social Sciences at the University of Western Australia. Her research revolves around considerations of digital media cultures, the governance of media infrastructures and the environmental impact of technological progress. https://research-repository.uwa.edu.au/en/persons/ katarina-damjanov

David Crouch is based in the School of Humanities at the University of Western Australia. His research engages with issues surrounding the material and social effects of media technologies, innovations in VR, robotics and the digital transformation of human experience. 
Roadsides is an open access journal designated to be a forum devoted to exploring the social, cultural and political life of infrastructure.

\section{Editorial Team:}

Julie Chu (University of Chicago)

Tina Harris (University of Amsterdam)

Agnieszka Joniak-Lüthi (University of Zurich)

Madlen Kobi (Academy of Architecture, Mendrisio)

Nadine Plachta (Heidelberg University's South Asia Institute, Kathmandu Office)

Galen Murton (LMU Munich and James Madison University, Harrisonburg)

Matthäus Rest (Max-Planck-Institute for the Science of Human History, Jena)

Alessandro Rippa (Tallinn University)

Martin Saxer (LMU Munich)

Christina Schwenkel (University of California, Riverside)

Max D. Woodworth (The Ohio State University)

Collection no. 003 was edited by: Christine Bichsel

Managing editor: Agnieszka Joniak-Lüthi

Copyediting: David Hawkins

Layout: Chantal Hinni and Antoni Kwiatkowski

\section{ISSN 2624-9081}

\section{Creative Commons License}

This work is licensed under a Creative Commons Attribution-NonCommercial-ShareAlike 4.0 International License.

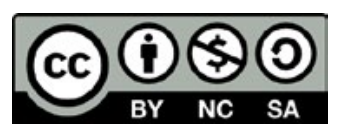

